\title{
Review of Jones-Wilkins-Lee equation of state
}

\author{
G. Baudin and R. Serradeill
}

CEA, DAM, 46500 Gramat, France

\begin{abstract}
The JWL EOS is widely used in different forms (two, three terms) according to the level of accuracy in the pressure-volume domain that applications need. The foundations of the relationship chosen to represent the reference curve, Chapman-Jouguet (CJ) isentrope, can be found assuming that the DP expansion isentrope issued from the $\mathrm{CJ}$ point is very nearly coincident with the Crussard curve in the pressure-material velocity plane. Its mathematical expression, using an appropriate relationship between shock velocity and material velocity leads to the exponential terms of the JWL EOS. It well validates the pressure-volume relationship chosen to represent the reference curves for DP. Nevertheless, the assumption of constant Gruneisen coefficient and heat capacity in the EOS thermal part remains the more restrictive assumption. A new derivation of JWL EOS is proposed, using a less restrictive assumption for the Gruneisen coefficient suggested by W.C. Davis to represent both large expansions and near-CJ states.
\end{abstract}

\section{Introduction}

The Jones-Wilkins-Lee [1] (JWL) equation of state (EOS) is widely used in reactive hydro simulations to describe thermodynamics of both detonation products (DP) and unreacted high explosives (HE). Its development follows earlier EOS proposed by Jones and Miller [2] and Wilkins [3]. It was referred as Jones-Wilkins-Lee equation in the reference [1] where it is completely described. This EOS is generally considered as a pure empirical EOS with a non physical Gruneisen coefficient considered constant. It is used in different forms (two, three terms) according to the level of accuracy in the pressure-volume domain that applications need. The earlier papers did not fully describe the basic assumptions of the pressure-volume relationship chosen to represent the reference curve, i.e. the Chapman-Jouguet (CJ) isentrope. The temperature can be evaluated adding constant heat capacity hypothesis. This paper thoroughly reviews the assumptions of the model and provides a derivation of JWL EOS based on a more physical assumption for the Gruneisen coefficient.

\section{On the choice of JWL isentrope}

Foundations of the relationship used for the JWL isentrope can be found using the following properties: (1) both the Rayleigh line, reactive shock Crussard curve and expansion isentrope, are tangent at the CJ point in the pressure-volume plane, (2) the DP expansion isentrope from any point on the Crussard curve is very nearly coincident with the Crussard curve in the pressure-material velocity (P-u) plane, (3) the pressure-material velocity relationship along the Crussard curve can be expressed as a universal curve in the P-u plane.

The first property is a consequence of the CJ model. The second property is frequently used to determine the thermodynamic state at detonation products-metal interface. In a similar way, W.C. Davis [4] developed a complete EOS for unreacted solid HE, using the property that the

This is an Open Access article distributed under the terms of the Creative Commons Attribution-Noncommercial License 3.0, which permits unrestricted use, distribution, and reproduction in any noncommercial medium, provided the original work is properly cited. 


\section{EPJ Web of Conferences}

expansion isentrope from any point of the Hugoniot curve is very nearly coincident with the Hugoniot curve in the $\mathrm{P}$-u plane. This property, applied for compression/release of inert material gives an exponential form EOS similar to exponential terms of the JWL EOS.

The last property has been proposed by Gimenez et al in 1988 for a large range of HE in the context of DP EOS development in the vicinity of CJ point [5]. The Crussard curve describes the fully reactive shock states from room pressure and temperature given by the equation $P=\rho_{0} D u$, the ambient pressure $P_{0}$ being neglected comparing to the pressure along the Crussard curve. At the CJ state, $P_{C J}=\rho_{0} D_{C J} u_{C J}$ and the ratio $P / P_{C J}$ reads

$$
\frac{P}{P_{C J}}=\frac{D}{D_{C J}} \frac{u}{u_{C J}}
$$

For a wide range of ideal HE, Gimenez et al demonstrated, using BKW thermochemical simulations, that the detonation velocity $\mathrm{D}$ is well represented by a "universal" relationship

$$
\frac{D}{D_{C J}}=a\left(\frac{u}{u_{C J}}\right)^{-1}+b+c\left(\frac{u}{u_{C J}}\right)
$$

Introduced in the previous equation, they obtained $\frac{P}{P_{C J}}=a+b\left(\frac{u}{u_{C J}}\right)+c\left(\frac{u}{u_{C J}}\right)^{2}$. In 1996, P.W. Cooper [6] proposed a similar "universal" $\mathrm{P}-\mathrm{u}$ relationship for the Crussard curve:

$$
\frac{P}{P_{C J}}=2.412-1.7315 \frac{u}{u_{C J}}+0.3195\left(\frac{u}{u_{C J}}\right)^{2}
$$

The first property is expressed by the relation $(\mathrm{d} P / \mathrm{d} v)_{S}=(\mathrm{d} P / \mathrm{d} v)_{C}$. The CJ isentrope of DP is very nearly coincident with the Crussard curve in the pressure-material velocity plane and $(\mathrm{d} P / \mathrm{d} v)_{S} \approx$ $(\mathrm{d} P / \mathrm{d} v)_{C}$ so that

$$
\frac{P_{S}}{P_{C J}} \approx a+b\left(\frac{u}{u_{C J}}\right)+c\left(\frac{u}{u_{C J}}\right)^{2} \text { along the CJ isentrope and } \frac{u_{C J}}{P_{C J}} \frac{\mathrm{d} P_{S}}{\mathrm{~d} u}=b+2 c\left(\frac{u}{u_{C J}}\right) .
$$

On an isentrope, $\left(\mathrm{d} P_{S} / \mathrm{d} u\right)^{2}=-\mathrm{d} P_{S} / \mathrm{d} v=-(\rho c)^{2}$. Thus, the previous equations lead to an ordinary differential equation which can be integrated to give

$P_{S}(v)=A \exp \left(-R \frac{v}{v_{0}}\right)+B$ with $A=\left(P_{C J}-P_{\infty}\right) e^{R}, B=P_{C J}\left(a-\frac{b^{2}}{4 a c}\right), R=4 c \frac{\rho_{C J}}{\rho_{0}}\left(\frac{\rho_{0} D_{C J}^{2}}{P_{C J}}-1\right)$.

This relation has an evident similarity with the first exponential term of JWL. The term $B$ represents a few percent of the pressure and can be neglected. The exponential term strongly decreases toward 0 for increasing DP expansion. Adding the ideal gas pressure-volume tendency ensures a correct behaviour at large expansion. Another refinement is to add a second exponential term to better reproduce the intermediate DP expansion:

$$
P_{S}(v)=A \exp \left(-R_{1} \frac{v}{v_{0}}\right)+B \exp \left(-R_{2} \frac{v}{v_{0}}\right)+C\left(\frac{v}{v_{0}}\right)^{-(\omega+1)}
$$

It is the well known JWL isentrope used in the Mie-Grunseisen formulation:

$$
e=e_{S}(v)+\frac{v}{\Gamma}\left(P-P_{S}(v)\right), \quad P_{S}(v)=-\left(\mathrm{d} e_{S}(v) / \mathrm{d} v\right), \quad \Gamma=\omega=\text { cste },
$$

$\Gamma=-(\partial \log T / \partial \log v)_{S}$ being the definition of the Gruneisen coefficient, $e_{S}(v)$ and $P_{S}(v)$ being the internal energy and pressure evolutions along the $\mathrm{CJ}$ isentrope.

This analysis well validates the pressure-volume JWL relationship chosen to represent the reference curves for DP. Nevertheless, the hypothesis of a constant Gruneisen coefficient remains the more restrictive assumption. 


\section{A new derivation of JWL EOS}

A new derivation of JWL EOS is proposed using a less restrictive assumption for the Gruneisen coefficient. An EOS must verify the convexity and the Bethe-Weyl criteria: $C_{v} \geq 0, c^{2} \geq 0, c^{2} /\left(C_{v} T\right) \geq \Gamma^{2}$, $\Gamma>0$ and $\left(\partial\left(\rho c^{2}\right) / \partial v\right)<0$ in the domain of interest. The JWL isentrope pressure-volume relationship is unchanged so that the adiabatic index $\gamma=-(\partial \log P / \partial \log v)_{S}$ will be also unchanged. The hypothesis of constant Gruneisen coefficient, is replaced by an assumption suggested by W.C. Davis [7] to represent both large expansions and near-CJ states:

$$
\Gamma(v)=\omega+\frac{2 \alpha}{\left(\frac{v}{v_{c}}\right)^{2 n}+1}
$$

$\gamma P / v, \quad-\Gamma T / v$ and $T / C_{V}$ represents the three second derivatives of the fundamental equation $e(v, s)$. Their modelling allows the construction of $e(v, s)$. The assumption $\Gamma=\Gamma(v)$ along with the thermodynamic equation $\frac{v}{C_{v}^{2}}\left(\frac{\partial C_{V}}{\partial v}\right)_{S}=\left(\frac{\partial \Gamma}{\partial s}\right)_{V}$ (invariance of the third derivation order of $e(v, s)$ ) show that $C_{V}$ must be a function of entropy s only. In a similar way to Davis' [4] for unreacted HE, it is assumed that $C_{V}=C_{V}^{C J}+\beta\left(s-s_{C J}\right)$ where $C_{V}^{C J}$ is the specific heat at the CJ detonation state. The integration of $\Gamma=-(\partial \log T / \partial \log v)_{S}$ at constant volume from the CJ point on the reference isentrope gives the temperature evolution $T_{S}(v)$ along the CJ isentrope. The form of $C_{V}(s)$ and the integration of thermodynamic equations $C_{V}=(\partial \log T / \partial v)_{S}$ along with the Maxwell relation $T=(\partial e / \partial s)_{V}$ lead to the following fundamental equation for the EOS:

$$
e(v, s)=e_{S}(v)+\frac{C_{V}^{C J} T_{S}(v)}{\beta+1}\left(\left(\frac{\beta\left(s-s_{C J}\right)}{C_{V}^{C J}}+1\right)^{\frac{\beta+1}{\beta}}-1\right)
$$

The functions $P(v, s), T(v, s)$ and the Mie-Gruneisen formulation $P(v, e)=P_{S}(v)+\frac{\Gamma(v)}{v}\left(e-e_{S}(v)\right)$ derives from this fundamental equation via the Maxwell thermodynamic equations $T=(\partial e / \partial s)_{V}$ and $P=(\partial e / \partial v)_{S}$. This model allows the computation of the DP entropy and temperature.

\section{Calibration and validation}

The classical method dedicated to JWL parameters calibration is the cylinder expansion test experiment. For applications that involve non-ideal detonation (curvature effects, presence of metallic particles) this method is not accurate and an alternative method is proposed, checking both detonation properties and curvature effects, necessary to guarantee that detonation has a physical wave structure. The CJ expansion isentrope of the organic HE part is fitted on a thermochemical calculation to avoid the curvature effects on the $\mathrm{CJ}$ detonation properties, the $\mathrm{CJ}$ model being established for plane detonation waves. The chemical rate law is thus adjusted to reproduce the curvature effects on detonation velocity.

This new derivation of JWL EOS provides a more physical evolution of the thermal part, compared to thermochemical code calculations: the evolutions of Gruneisen coefficient and heat capacity versus specific volume and entropy along the Crussard curve and the main isentrope are well reproduced. Plane sustained overdriven detonations provide constant states easily obtained inside nitromethane (NM) by plane impact experiments at pressures higher than the CJ pressure in DP. Some experimental and calculated results are presented Fig. 1 to illustrate the capability of our derivation of JWL EOS. The DP EOS is here calibrated on a BKWC calculation using Cheetah 2.0.

\section{Conclusion}

Our derivation of JWL EOS provides a more physical evolution of the thermal part, compared to thermochemical code calculations. The mathematical form obtained is expressed as a complete EOS 
EPJ Web of Conferences
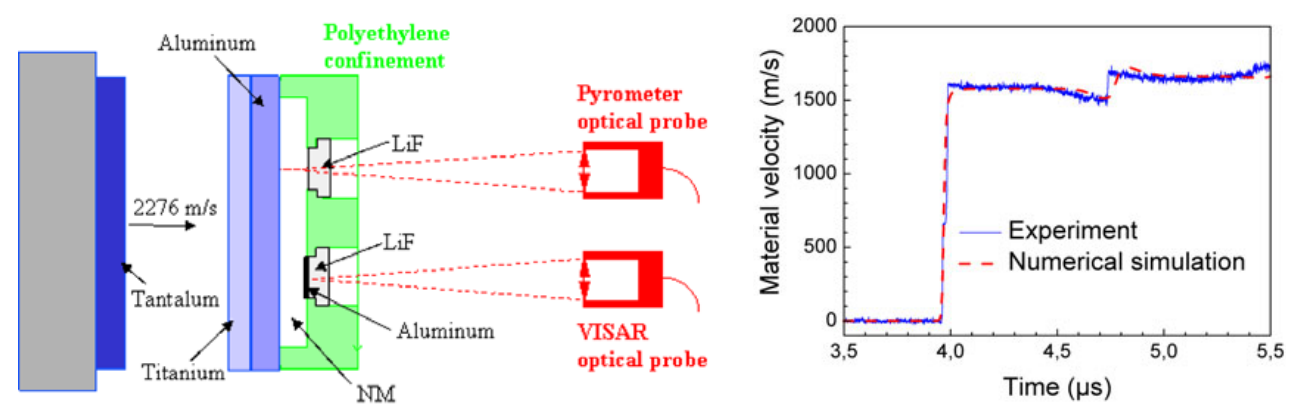

Fig. 1. Overdriven detonation in nitromethane at $19.2 \mathrm{GPa}$.

and allows the calculation of temperature using a heat capacity depending on entropy according to the thermodynamic relations. The similarity of the EOS forms for DP and unreacted explosive (Davis [4]) is useful for implementation in hydrocodes. This EOS has been calibrated for several conventional solid high explosives.

However, it is clear that an equation of state calibrated for DP expansions less than 10-20 times the initial volume can have severe limits at large expansion in ambient air where occurs a secondary combustion that makes it difficult to calibrate. Presently, the validity of such an EOS in the whole domain of expansion is not known because of the lack of experimental data at large expansion. However, such an EOS allows calculations of HE detonation and metal expansion in classic charges.

\section{References}

1. E.L. Lee, H.C. Horning, J.W. Kury, Adiabatic expansion of high explosives detonation products, Lawrence Livermore National Laboratory, University of California, Livermore, TID 4500-UCRL 50422 (1968).

2. H. Jones, A.R. Miller, The detonation of solid explosives, Proc. Roy. Soc. London, A-194, 480 (1948)

3. M.L. Wilkins, The equation of state of PBX 9404 and LX04-01, Lawrence Radiation Laboratory, Livermore, report UCRL-7797 (1964).

4. W.C. Davis, Complete Equation of State for Unreacted Solid Explosive, Combustion and Flame 120:399-403 (2000).

5. P. Gimenez, D. Bergues, G. Baudin, DGA/CEG report (1988).

6. P.W. Cooper, Explosives Engineering, WILEY-VCH (1996).

7. W.C. Davis, Equation of state for detonation products, 10th International Symposium on Detonation, 369-384 (1993). 